\title{
miR-135a acts as a tumor suppressor in gastric cancer in part by targeting KIFCI
}

This article was published in the following Dove Press journal:

OncoTargets and Therapy

15 June 2016

Number of times this article has been viewed

\author{
Chuanlei Zhang' \\ Xiaoqi Chen' \\ Xinju Chen' \\ Xinting Wang' \\ Aiying $\mathrm{Ji}^{1}$ \\ Lifeng Jiang ${ }^{2}$ \\ Feng Sang ${ }^{3}$ \\ Fucheng $\mathrm{Li}^{4}$ \\ 'Department of Digestive Diseases, \\ The First Affiliated Hospital of \\ Henan University of Traditional \\ Chinese Medicine, ${ }^{2}$ Department of \\ Chinese Traditional and Western \\ Medicine, Henan Cancer Hospital, \\ Affiliated Cancer Hospital of \\ Zhengzhou University, ${ }^{3}$ Department \\ of Acquired Immune Deficiency \\ Syndrome Treatment and Research \\ Center, Key Laboratory of Viral \\ Diseases Prevention and Treatment \\ of Traditional Chinese Medicine of \\ Henan Province, ${ }^{4}$ The First Affiliated \\ Hospital of Henan University of \\ Traditional Chinese Medicine, \\ Zhengzhou, People's Republic \\ of China
}

\begin{abstract}
R-135a was downregulated in the majority of human primary gastric cancer (GC) tissues and GC cell lines. Kinesin family member C1 (KIFC1) was significantly upregulated in GC tissues and cell lines and promoted GC development and progression. We searched for miR-135a targets by using MiRanda, TargetScan, and PicTar tools, and found that KIFC1 was a potential target of miR-135a. Based on these findings, we speculated that miR-135a might target KIFC1 to inhibit GC growth. We determined the expression of miR-135a and KIFC1 by quantitative real-time polymerase chain reaction and Western blot assays, respectively, and found downregulation of miR-135a and upregulation of KIFC1 in GC tissues and cell lines. Cell proliferation and apoptosis assays showed that knockdown of KIFC1 inhibited proliferation and promoted apoptosis of GC cells, and miR-135a mimics had similar effects on GC cell proliferation and apoptosis. Furthermore, we verified that KIFC1 was a direct target of miR-135a, which confirmed our speculation that the functional effect of miR-135a on GC cells, at least, in part, depends on KIFC1. These findings suggest that miR-135a has an important role in the suppression of GC and presents a novel mechanism of miRNA-mediated KIFC1 expression in cancer cells.
\end{abstract}

Keywords: gastric cancer, miR-135a, kinesin family member C1, proliferation, apoptosis

\section{Introduction}

Gastric cancer (GC) is one of the most common malignancies, and is also the leading cause of cancer-related mortalities. ${ }^{1,2}$ Despite achieving progress in the development of new management strategies, GC remains difficult to diagnose at an early stage. Most GC patients experience asymptomatic presentation in the early stage, resulting in metastasis at diagnosis, and the clinical outcome of advanced GC patients is extremely poor. ${ }^{3}$ To improve the clinical outcome of GC treatment, it is important to clarify the molecular pathogenesis of GC and investigate the genes responsible for GC development and progression.

MicroRNAs (miRNAs) are an evolutionarily conserved group of small noncoding RNAs that posttranscriptionally regulate gene expression and play important roles in a variety of physiological and pathological processes, such as development, cell proliferation, apoptosis, differentiation, and signal transduction. ${ }^{4}$ To date, more than 1,000 human miRNAs have been identified. ${ }^{5}$ Increasing evidence has shown that miRNAs are frequently dysregulated in human malignant tumors and that miRNAs can act as oncogenes or tumor suppressor genes, which depend on the genes they modulate. ${ }^{6-10}$ For example, miR-21 downregulates tumor suppressor Pdcd4 and stimulates invasion, intravasation, and metastasis in colorectal cancer; ${ }^{6} \mathrm{miR}-7$ suppresses brain metastasis of breast cancer stem-like cells by regulating KLF $4 ;{ }^{7}$ miR-103/107 promotes metastasis 
of colorectal cancer by repressing the metastasis suppressors DAPK and KLF4; ${ }^{8}$ miR-34a inhibits proliferation and migration of breast cancer through downregulation of B-cell lymphoma 2 (Bcl-2) and SIRT1; 9 and miR-218 functions as a tumor suppressor in head and neck squamous cell carcinoma by inhibiting cell migration and invasion. ${ }^{10}$ Recently, a substantial number of deregulated miRNAs have been reported to be involved in cell proliferation, apoptosis, invasion, and migration in GC. For example, miR-218 inhibits invasion and metastasis of GC by targeting the Robol receptor; ${ }^{11}$ miR-375 is frequently downregulated in GC and inhibits cell proliferation by downregulating JAK2 ${ }^{12}$ miR-150 promotes GC proliferation by negatively regulating the proapoptotic gene $E G R 2 ;{ }^{13}$ and miR-217 inhibits tumor progression and metastasis by targeting EZH2 and predicts favorable prognosis in $\mathrm{GC}{ }^{14}$

In recent years, miR-135a has been widely studied due to its controversial role in cancers. For example, miR-135a is upregulated and contributes to the development of portal vein tumor thrombus by promoting metastasis in hepatocellular carcinoma, ${ }^{15}$ enhances cellular proliferation through regulation of PHLPP2 and FOXO1 in human bladder cancer, ${ }^{16}$ and promotes growth and invasion of colorectal cancer via regulation of metastasis suppressor $1,{ }^{17}$ whereas miR-135a is downregulated and functions as a selective killer of malignant glioma, ${ }^{18}$ acts as a tumor suppressor in epithelial ovarian cancer and regulates HOXA10 expression, ${ }^{19}$ inhibits cancer cell proliferation by targeting the c-MYC oncogene in renal cell carcinoma, ${ }^{20}$ and suppresses migration and invasion and regulates epithelial-mesenchymal transition-related marker genes by targeting KLF8 in lung cancer cells. ${ }^{21}$ These controversial results may reflect the diverse roles of miR-135a in different forms of cancer. Recently, miR$135 \mathrm{a}$ has been found to be downregulated in GC through analysis of a published microarray-based high-throughput assessment. ${ }^{22}$ We used MiRanda, TargetScan, and PicTar tools to perform bioinformatics-based target prediction analysis, and found that JAK2 and kinesin family member C1 (KIFC1) were potential targets of miR-135a. KIFC1 has been widely reported to be upregulated in various cancers, such as ovarian cancer, non-small-cell lung cancer, and breast cancer, and promotes the initiation and progression of cancer. ${ }^{23-25} \mathrm{Wu}$ et $\mathrm{al}^{26}$ have reported that miR-135a targets JAK2 and inhibits GC cell proliferation. Recently, KIFC1 has been reported to be upregulated in GC cells grown as spheroids and GC tissues, and promotes GC development and progression. ${ }^{27}$ Therefore, we speculate that miR-135a might regulate $\mathrm{GC}$ cell proliferation and apoptosis in part by targeting $\mathrm{KIFC} 1$.
In this study, we examined the expression level of miR-135a and $\mathrm{KIFC} 1$ in GC tissues and cell lines by quantitative realtime polymerase chain reaction (qRT-PCR) and Western blot, respectively, and investigated the association between miR-135a and KIFC1 expression and GC cell proliferation and apoptosis. We conducted dual-luciferase reporter assay to identify whether the $3^{\prime}$-untranslated region (3'-UTR) of KIFC1 messenger RNA (mRNA) is a binding target of miR-135a. These findings will provide new clues for the development and progression of GC and promising therapeutic targets in the treatment of GC.

\section{Materials and methods}

\section{Patients and tissue specimens}

A total of 15 paired GC tissues and adjacent nontumor tissues were obtained from patients with primary GC undergoing radical gastrectomy at The First Affiliated Hospital of Henan University of Traditional Chinese Medicine, People's Republic of China, from May 2013 to August 2015. All these patients did not receive any preoperative treatment, such as radiotherapy or chemotherapy. After surgical removal, the tissues were snap-frozen in liquid nitrogen and stored at $-80^{\circ} \mathrm{C}$. This study was approved by the Ethics Review Board of the The First Affiliated Hospital of Henan University of Traditional Chinese Medicine, and the written informed consents were signed by all patients before participation.

\section{Cell culture and transfection}

Human GC cell lines AGS, BGC-823, MKN-28, MKN-45, MGC-803, and SGC-7901 and human embryonic kidney 293 (HEK-293) cells were purchased from the Shanghai Institutes for Biological Sciences (Shanghai, People's Republic of China) and routinely cultured in Roswell Park Memorial Institute 1640 medium (Invitrogen, Carlsbad, CA, USA) supplemented with 10\% fetal bovine serum (HyClone, Logan, UT, USA), $100 \mathrm{U} / \mathrm{mL}$ penicillin, and $100 \mu \mathrm{g} / \mathrm{mL}$ streptomycin. All cells were maintained in a humidified incubator containing $5 \%$ carbon dioxide at $37^{\circ} \mathrm{C}$.

The miR-135a mimics, miR-135a inhibitors, smallinterfering RNAs targeting KIFC1 (siKIFC1), short-hairpin RNA expression vectors targeting KIFC1 (shKIFC1) and their respective controls were obtained from GenePharma (Shanghai GenePharma Co. Ltd, Shanghai, People's Republic of China). The cells were seeded into six-well plates, incubated for 24 hours, and then transfected with miR-135a mimics, inhibitors, siKIFC1, or shKIFC1 by using Lipofectamine 2000 (Invitrogen), in accordance with the manufacturer's suggestions. At 48 hours posttransfection, cells were harvested and used in subsequent experiments. 


\section{Quantitative real-time PCR}

Total RNA was isolated from frozen GC tissues or cultured cells by using Trizol agent (Invitrogen) following the manufacturer's instructions. The expression level of miR-135a was determined by using TaqMan miRNA assays (Ambion, Austin, TX, USA) with miRNA-specific primers (forward primer: 5'-ACA CTC CAG CTG GGT ATG GCT TTT TAT TCC T- $3^{\prime}$ and universal reverse primer: 5'-GGT GTC GTG GAG TCG GCA A-3'), and U6 small nuclear RNA was used as an endogenous control for data normalization. The expression level of KIFC1 mRNA was determined by using PrimeScript RT-PCR kits (Takara Biochemicals, Kyoto, Japan) with primers (forward primer: 5'-GGT CGT ATT GGG CGC CTG GTC-3' and backward primer: 5'-TGA CGG TGC CAT GGA ATT TGC CA-3'), and glyceraldehyde-3-phosphate dehydrogenase was used as an internal control.

\section{Cell proliferation assay}

Cell proliferation was monitored by the colorimetric watersoluble tetrazolium salt assay using a cell counting kit-8 (Dojindo, Kumamoto, Japan), following the manufacturer's instructions. Cells with $2 \times 10^{3}$ cells per well were seeded in 96-well plates, and cell proliferation was documented every 24 hours for a period of 4 days. The number of viable cells was assessed by determining the absorbance at $450 \mathrm{~nm}$ using a monochromator microplate reader (Safire II, Tecan, Switzerland).

\section{Cell apoptosis assay}

An annexin V-fluorescein isothiocyanate apoptosis detection kit I (BD Pharmingen, San Diego, CA, USA) was used in the apoptosis assay in accordance with the manufacturer's instructions. Briefly, cells were washed in phosphate-buffered saline and resuspended in $1 \times$ binding buffer at a concentration of $1 \times 10^{6}$ cells $/ \mathrm{mL}$; then, $5 \mu \mathrm{L}$ of annexin V-fluorescein isothiocyanate and $5 \mu \mathrm{L}$ of propidium iodide were added to $100 \mu \mathrm{L}$ of cell suspension and the samples were incubated in darkness; after incubation for 15 minutes, $400 \mu \mathrm{L} 1 \times$ binding buffer was added to the samples. Apoptosis was analyzed by flow cytometry (BD Biosciences, San Jose, CA, USA) using the CellQuest software (BD Biosciences).

\section{Western blot assay}

Total proteins were extracted from frozen GC tissues or cultured cells using radioimmunoprecipitation assay lysis buffer $(50 \mathrm{mmol} / \mathrm{L}$ Tris-HCl $\mathrm{pH} 7.4 ; 0.2 \%$ sodium dodecyl sulfate; $0.2 \%$ sodium deoxycholate; $1 \mathrm{mmol} / \mathrm{L}$ ethylenediaminetetraacetic acid; $1 \%$ Triton X-100). Total proteins were isolated and resolved by $10 \%$ sodium dodecyl sulfate polyacrylamide gel electrophoresis and then transferred to polyvinylidene difluoride membranes (BioRad, Hercules, CA, USA). The membranes were probed with primary antibodies overnight, followed by incubation with horseradish peroxidase-conjugated secondary antibodies. Protein expression was assessed with a chemiluminescence kit (Amersham Life Science, Buckingham, UK). The signal intensity of the protein bands was quantified by using Quantity One 4.5.0 software (BioRad). Anti-KIFC1, anti-Bax, and anti-Bcl-2 antibodies were purchased from Abcam (Cambridge, UK), and $\beta$-actin (Abcam) was used as an internal control.

\section{Luciferase reporter assay}

The fragment of wild-type KIFC1 3'-UTR (3'-UTR-WT) containing predicted miR-135a target sites was amplified by PCR, and mutant KIFC1 3'-UTR (3'-UTR-MUT) was generated by mutating the conserved binding sites for miR-135a using overlap-extension PCR method. The fragments including the 3'-UTR-WT or 3'-UTR-MUT regions of KIFC1 were cloned into XhoI/NotI-digested psiCHECK-2 vector (Promega, Madison, WI, USA), which included both renilla and firefly luciferase reporter genes. Then the psiCHECK-2 vectors with 3'-UTR-WT or 3'-UTR-MUT regions of KIFC1 were transfected into MKN-45 and MGC-803 cells transfected with miR-135a mimics, negative control mimics, miR-135a inhibitors, or negative control inhibitors, respectively. After 24 hours, the cells were harvested, and the firefly and renilla luciferase activities were determined with a dual-luciferase reporter assay system (Promega Corporation, Fitchburg, WI, USA) in accordance with the manufacturer's suggestions.

\section{Statistical analysis}

All statistical analyses were performed using GraphPad prism 5 (GraphPad Software Inc., San Diego, CA, USA). The results were presented as mean \pm standard deviation. Differences between groups were examined for statistical significance using the Student's $t$-test, and a two-tailed value of $* P<0.05, * * P<0.01$, or $* * * P<0.001$ was, in all cases, considered statistically significant.

\section{Results}

\section{miR-I35a is frequently downregulated and $\mathrm{KIFCI}$ is upregulated in GC}

The expression levels of miR-135a and KIFC1 mRNA in 15 paired GC tissues and adjacent nontumor tissues were detected by qRT-PCR. Compared with the nontumor tissues, the GC tissues showed downregulation of miR-135a and 
upregulation of KIFC1 mRNA (Figure 1A and B). A negative correlation was observed between the expression levels of miR-135a and FOXM1 mRNA in tumor samples $(R=-0.819$, Figure 1C). The mean KIFC1 protein level was significantly higher in GC tissues than that in matched nontumor tissues (Figure 1D). In addition, the expression levels of miR-135a and KIFC1 in GC cell lines were also detected. As shown in Figure 1E-G, miR-135a level was significantly downregulated in GC cell lines compared to that in HEK-293 cells, and the expression levels of KIFC1 mRNA and protein were significantly upregulated in GC cell lines compared to that in
HEK-293 cells. These results indicated that downregulation of miR-135a and upregulation of KIFC1 exist in GC.

\section{Knockdown of KIFCI inhibits proliferation and promotes apoptosis of GC cells}

To examine the role of KIFC1, endogenous KIFC1 expression was modulated in MKN-45 and MGC-803 cells by RNA interference strategies. Results showed that MKN-45 and MGC-803 cells transfected with shKIFC1 or siKIFC1, had significant decreases in KIFC1 expression compared
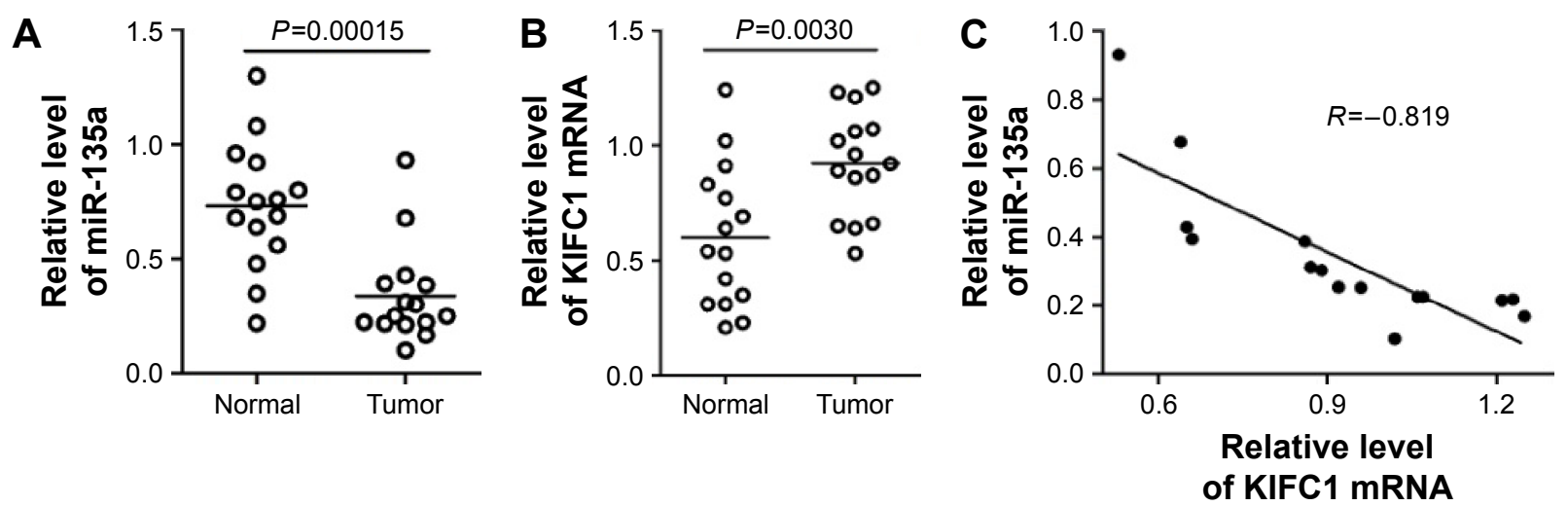

D
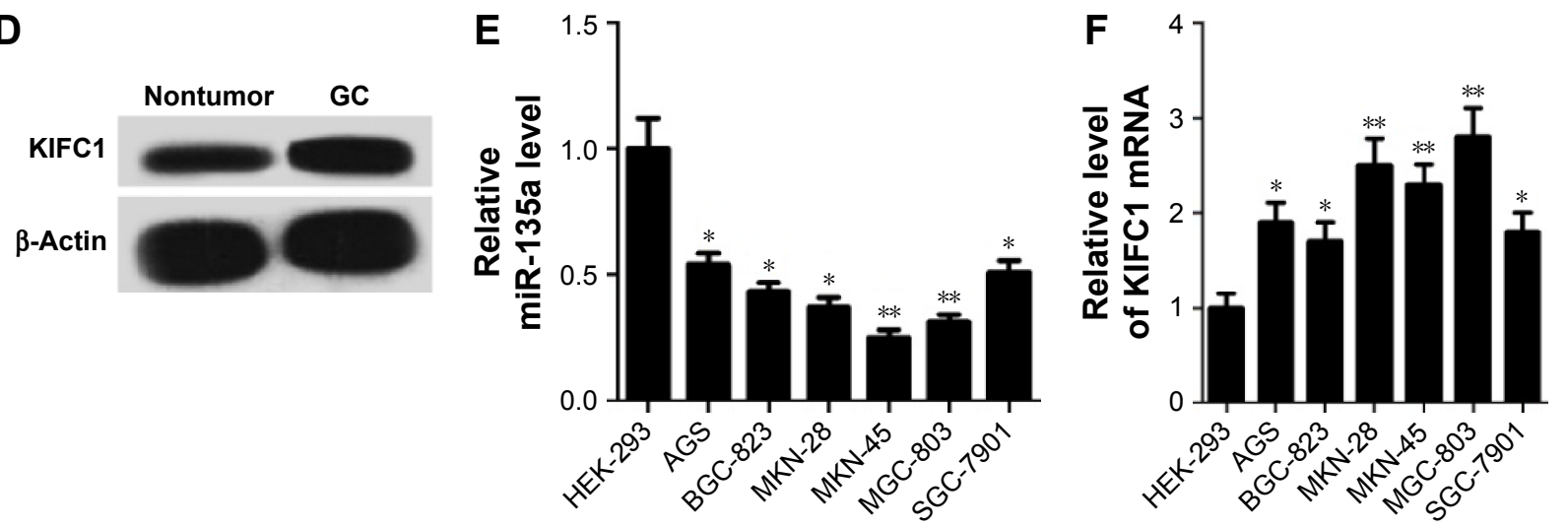

G

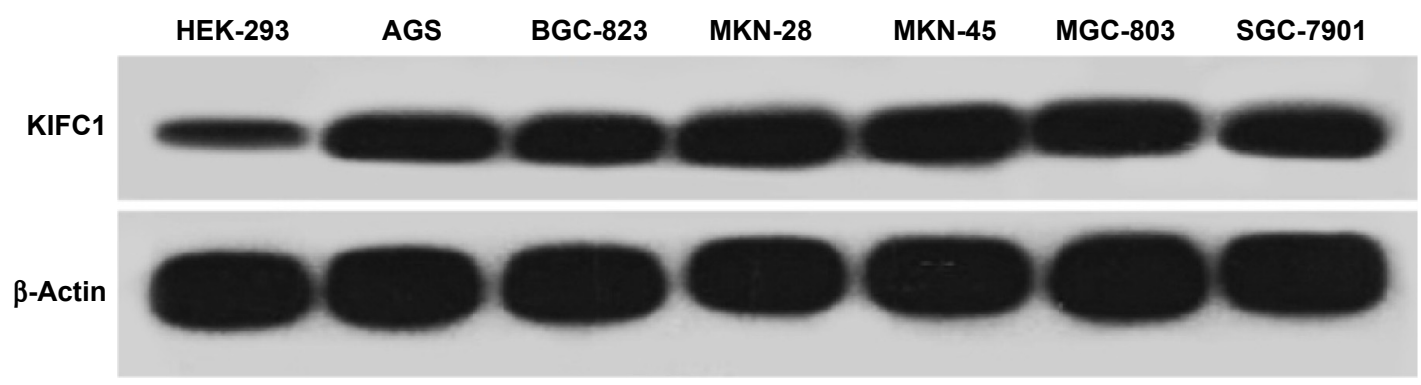

Figure I miR-I35a is downregulated and KIFCI is upregulated in GC.

Notes: (A and B) qRT-PCR shows that miR-I35a is frequently downregulated and KIFCI is upregulated in GC tissues compared with their adjacent normal tissues. (C) A negative correlation is observed between the expression levels of miR-I35a and FOXMI mRNA in tumor samples ( $R=-0.819$ ). (D) Western blot assay shows that the mean KIFCI level is significantly higher in GC tissues than that in matched nontumor tissues. (E and F) qRT-PCR shows that miR-I35a level is downregulated and KIFCI mRNA level is significantly upregulated in GC cell lines compared with that in HEK-293 cells. (G) Western blot assay shows that KIFCI protein level is significantly upregulated in GC cell lines compared with that in HEK-293 cells. $* P<0.05$ and $* * p<0.01$.

Abbreviations: GC, gastric cancer; HEK, human embryonic kidney; mRNA, messenger RNA; qRT-PCR, quantitative real-time polymerase chain reaction. 
with controls (Figure 2A and B). Then we performed cell proliferation and apoptosis detection on MKN-45 and MGC803 cells transfected with shKIFC1, siKIFC1, short-hairpin RNA control (sh-control), or small-interfering RNA control (si-control) to investigate the effect of KIFC1 expression level on proliferation and apoptosis of GC cells. Results of the cell proliferation assays revealed that MKN-45 and MGC-803 cells transfected with shKIFC1 or siKIFC1 proliferated more slowly than those transfected with sh-control or si-control (Figure 2C and D). Results of cell apoptosis analysis revealed that knockdown of KIFC1 led to an increase in apoptosis of MKN-45 and MGC-803 cells (Figure 2E and F). In addition,
MKN-45 and MGC-803 cells transfected with shKIFC1 or siKIFC1 had significant increases in Bax expression and marked decreases in Bcl-2 expression (Figure 2G and $\mathrm{H}$ ). These results indicated that knockdown of KIFC1 inhibits proliferation and promotes apoptosis of GC cells.

\section{miR-I35a represses proliferation and promotes apoptosis of GC cells}

To investigate the effect of miR-135a on GC cell proliferation, we transfected MKN-45 and MGC-803 cells with miR-135a mimics, negative control mimics, miR-135a inhibitors, or negative control inhibitors and then performed
A

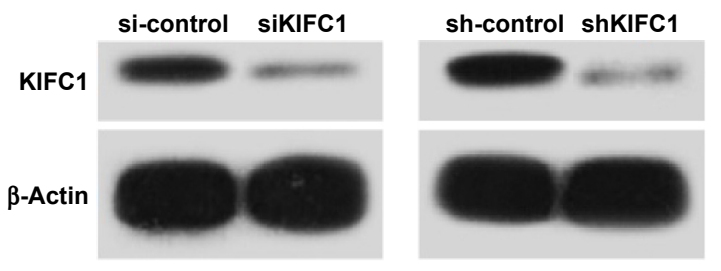

MKN-45

C
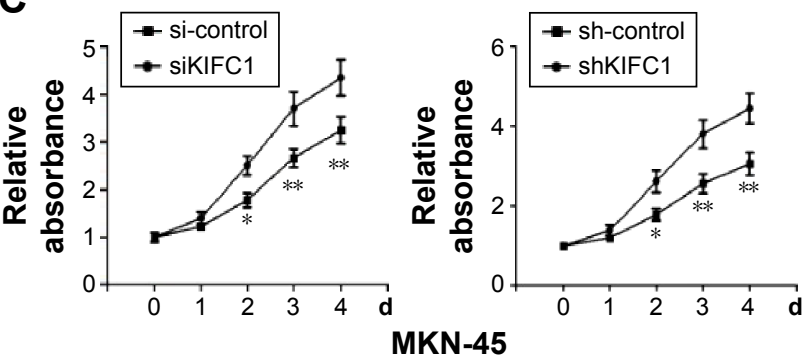

E
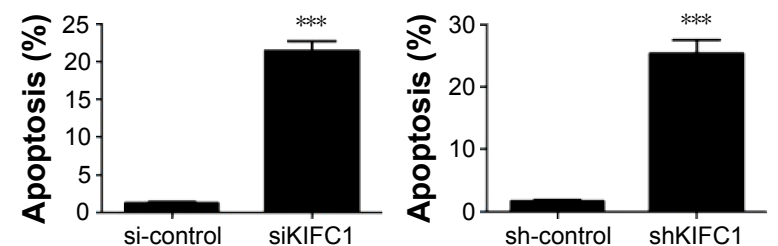

MKN-45

G

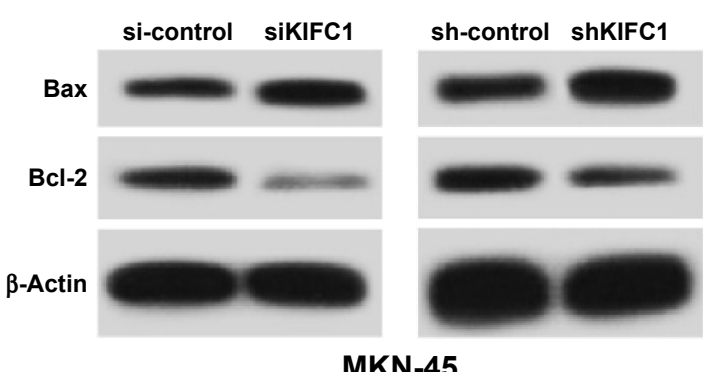

B

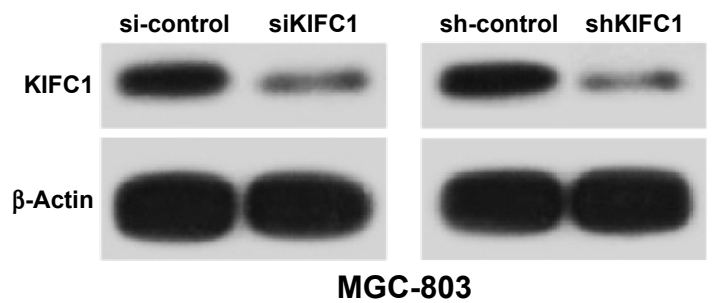

D
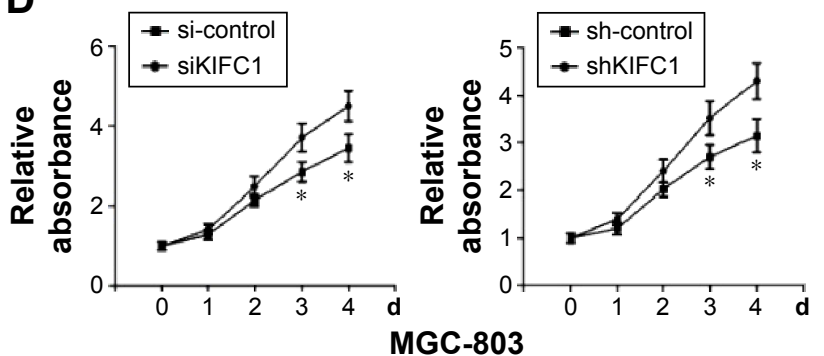

$\mathbf{F}$

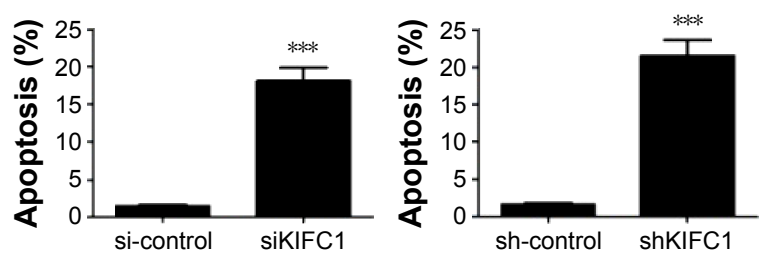

MGC-803

H

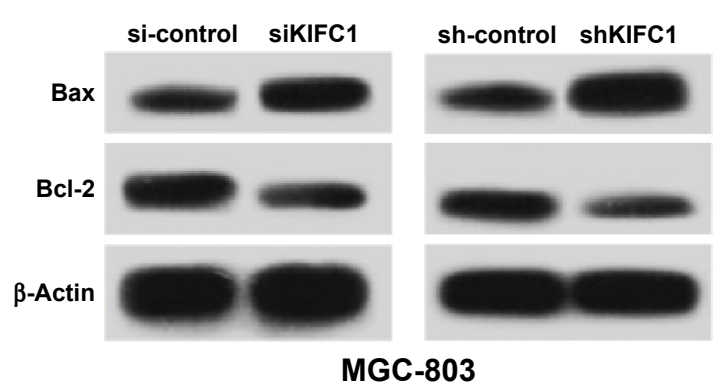

Figure 2 Knockdown of KIFCI inhibits proliferation and promotes apoptosis of GC cells.

Notes: (A and B) Western blot assay shows that MKN-45 and MGC-803 cells transfected with shKIFCI or siKIFCI, have significant decreases in KIFCI expression compared with controls. (C and D) Cell proliferation assay shows that MKN-45 and MGC-803 cells transfected with shKIFCI or siKIFCI proliferate slower than those transfected with sh-control or si-control. (E and F) Cell apoptosis assay shows that knockdown of KIFCI leads to a significantly increased apoptosis in MKN-45 and MGC-803 cells. ( $\mathbf{G}$ and $\mathbf{H}$ ) Western blot assay shows that MKN-45 and MGC-803 cells transfected with shKIFCI or siKIFCI have significant increases in Bax expression and decreases in $\mathrm{Bcl}-2$ expression. $* P<0.05$, $* * P<0.01$, and $* * * P<0.001$.

Abbreviations: GC, gastric cancer; sh-control, short-hairpin RNA control; si-control, small-interfering RNA control. 
cell proliferation detection. qRT-PCR showed that both MKN-45 and MGC-803 cells transfected with miR-135a mimics had higher miR-135a expression than the cells transfected with negative control mimics, and both MKN-45 and MGC-803 cells transfected with miR-135a inhibitors had lower miR-135a expression than the cells transfected with negative control inhibitors (Figure $3 \mathrm{~A}$ and $\mathrm{B}$ ). Cell proliferation assay revealed that both MKN-45 and MGC-803 cells, when transfected with miR-135a mimics, proliferated obviously slower than those transfected with negative control mimics (Figure 3C). In contrast, cells transfected with miR-135a inhibitors proliferated significantly faster than those transfected with negative control inhibitors (Figure 3D). We also performed apoptosis detection on MKN-45 and MGC-803 cells transfected with miR-135a mimics or negative control mimics. As shown in Figure 3E and F, MKN-45 and MGC-803 cells transfected with miR135 a mimics both had significantly higher apoptosis than controls. In addition, we performed Western blot assay to detect the expression level of apoptosis-related proteins Bax and Bcl-2, and found that both MKN-45 and MGC-803 cells transfected with miR-135a mimics had a significant increase in Bax expression and an obvious decrease in Bcl-2 expression (Figure $3 \mathrm{G}$ and $\mathrm{H}$ ). These results indicated that miR-135a inhibited proliferation and promoted apoptosis of GC cells. Similar effect of miR-135a mimics was observed in shKIFC1- and siKIFC1-treated cells, which suggested that miR-135a could mimic the effect of shKIFC1 and siKIFC1.

\section{$\mathrm{KIFCI}$ is a direct target of miR-I35a}

We searched for miR-135a targets by using MiRanda, TargetScan, and PicTar tools, and results identified KIFC1 as a putative target of miR-135a (Figure 4A). Then dual-luciferase reporter assay was performed on MKN-45 and MGC-803 cells to identify whether the $3^{\prime}$-UTR of KIFC1 mRNA is a binding target of miR-135a. MKN-45 and MGC-803 cells cotransfected with plasmid containing 3'-UTR-WT regions of KIFC1, and miR-135a mimics had significantly less luciferase activity than their controls, while mutation of the potential miR-135a binding sites in the KIFC1 3'-UTR abolished this effect (Figure 4B). In contrast, MKN-45 and MGC-803 cells cotransfected with plasmid containing 3'-UTR-WT regions of KIFC1 and miR-135a inhibitors had significantly higher luciferase activity than their controls, which was abrogated by mutating the tentative miR-135a binding sites in KIFC1 (Figure 4C). qRT-PCR and Western blot assays revealed that overexpression of miR-135a inhibited KIFC1 mRNA and protein expression in MKN-45 and MGC-803 cells (Figure 4D and E). In contrast, downregulation of miR-135a enhanced KIFC1 mRNA and protein expression in MKN-45 and MGC-803 cells (Figure 4F and G). Taken together, these results suggested that miR-135a directly targets KIFC1 in GC cells and the functional effect of miR-135a on GC cells, at least, in part, depends on its direct target KIFC1.

\section{Discussion}

Increasing evidence has indicated that dysregulation of miR-135a results in the development of cancers. GC is one of the most common cancers worldwide, especially in several Asian countries, including the People's Republic of China. ${ }^{28}$ In spite of achieving significant progress in the treatment of this type of malignancy, the prognosis of GC patients in advanced stages remains rather poor. ${ }^{3}$ Therefore, understanding the genetic and epigenetic alterations underlying GC development and progression is still needed. Many deregulated miRNAs have been reported to be involved in GC development and progression, including miR-135a. ${ }^{11-14}$ $\mathrm{Wu}$ et $\mathrm{al}^{26}$ reported that miR-135a was downregulated in the majority of human primary GC tissues and cell lines, and may function as a tumor suppressor by targeting JAK to suppress p-STAT3 activation, reduce cyclin D1 and Bcl- $\mathrm{x}_{\mathrm{L}}$ expression, and repress GC cell proliferation. We further investigated whether miR-135a exerted regulatory effects on GC cell proliferation and apoptosis by targeting other genes. We used MiRanda, TargetScan, and PicTar to perform bioinformatics-based target prediction analysis, and found that kinesin-like protein KIFC1 was also a potential target of miR-135a. KIFC1, a normally nonessential kinesin motor, plays a crucial role in centrosome clustering in cancer cells and is essential for cancer cell survival. Pawar et $\mathrm{al}^{23}$ reported that KIFC1 predicts worse prognosis, poor overall survival, and may act as a potential marker for onset of metastatic dissemination in ovarian cancer patients. Grinberg-Rashi et $\mathrm{al}^{24}$ reported that overexpression of KIFC1 together with two other proteins, fetal Alzheimer antigen and N-cadherin, is highly predictive of brain metastasis in early and advanced patients with non-small-cell lung cancer. Li et $\mathrm{al}^{25}$ found that KIFC1 expression is upregulated in breast cancer tissues and cell lines, and KIFC1 silencing reduces breast cancer cell viability. Recently, KIFC1 was reported to be upregulated in GC tissues and cell lines, and promoted GC development and progression. ${ }^{27}$ Therefore, we speculate that miR-135a might regulate GC cell proliferation and apoptosis in part by targeting KIFC1. In this study, we detected the expression levels of miR-135a and KIFC1 in GC tissues and cell lines, and found 
A

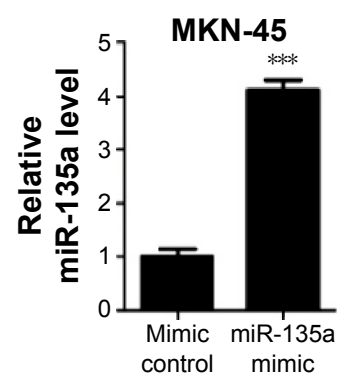

C

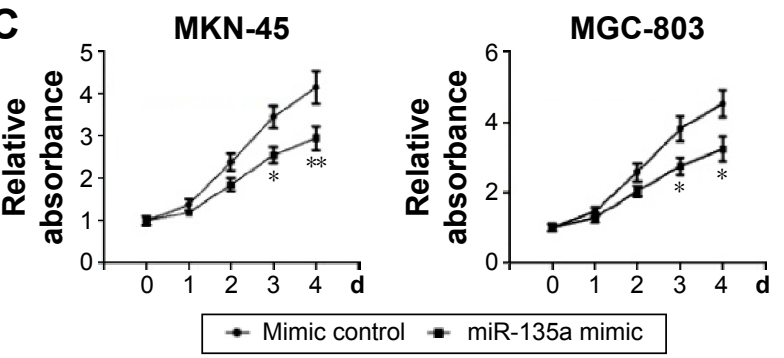

E

$\overline{\mathbf{L}}$

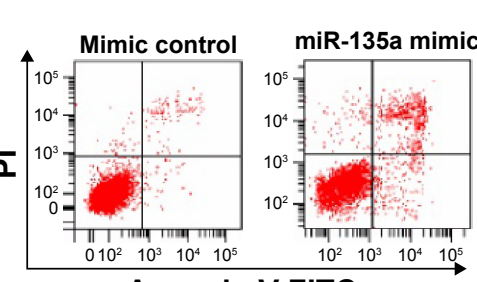

Annexin V-FITC

\section{G}
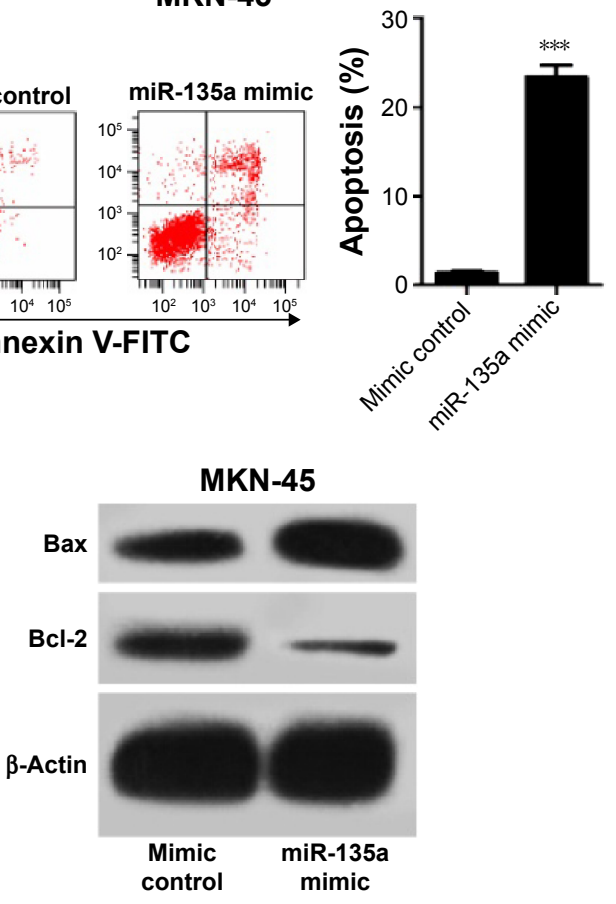
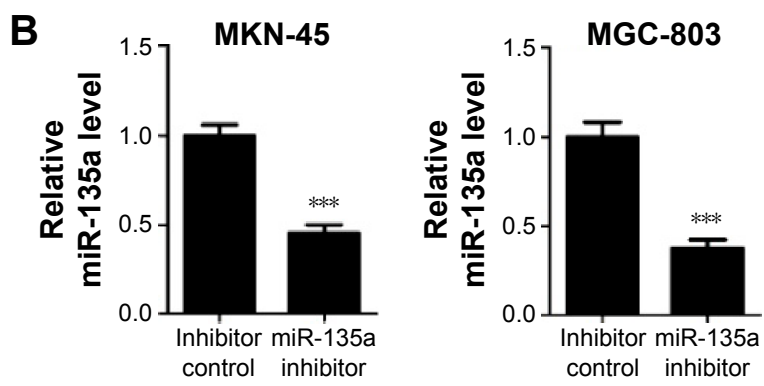

D

MKN-45

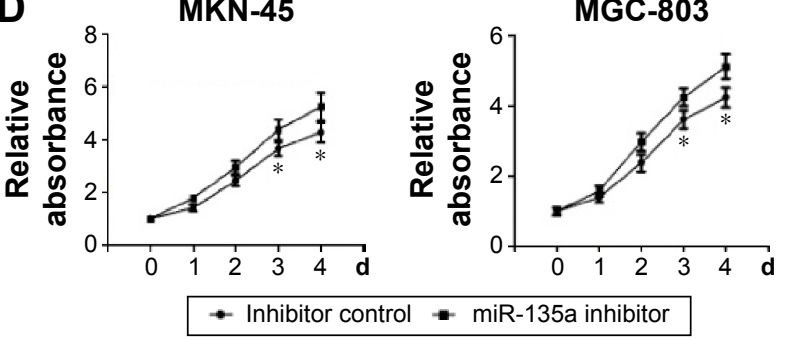

$\mathbf{F}$

$\overline{2}$

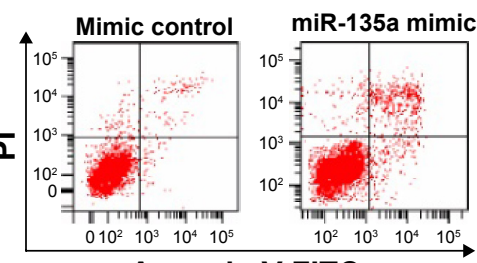

Annexin V-FITC

MGC-803

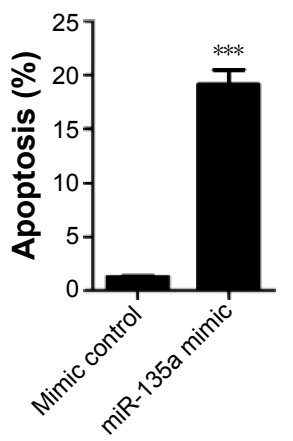

H

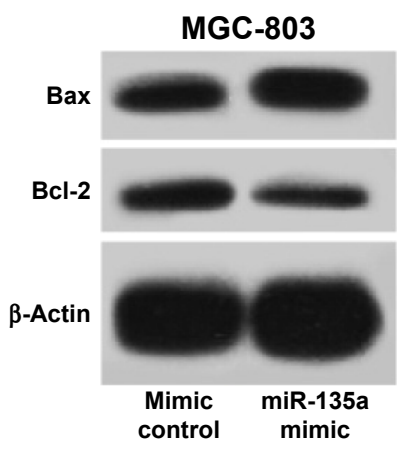

Figure 3 miR-135a inhibits proliferation and promotes apoptosis in GC cells.

Notes: (A) qRT-PCR shows that the expression level of miR-135a is higher in MKN-45 and MGC-803 cells transfected with miR-I35a mimics than in MKN-45 and MGC-803 cells transfected with negative control mimics. (B) qRT-PCR shows that the expression level of miR-135a is lower in MKN-45 and MGC-803 cells transfected with miR-I35a inhibitors than in MKN-45 and MGC-803 cells transfected with negative control inhibitors. (C) Cell proliferation assay shows that MKN-45 and MGC-803 cells transfected with miR-135a mimics proliferate more slowly than those transfected with negative control mimics. (D) Cell proliferation assay shows that MKN-45 and MGC-803 cells transfected with miR-135a inhibitors proliferate significantly faster than those transfected with negative control inhibitors. (C, E, and $\mathbf{F}$ ) Cell apoptosis assay shows that MKN-45 and MGC-803 cells transfected with miR-135a mimics both have significantly higher apoptosis than controls. (G and $\mathbf{H})$ Western blot assay shows that MKN-45 and MGC-803 cells transfected with miR-135a mimics both have a significant increase in Bax expression and an obvious decrease in Bcl-2 expression compared with the cells transfected with negative control mimics. $* P<0.05$, $* * P<0.01$ and $* * * P<0.001$.

Abbreviations: GC, gastric cancer; $\mathrm{QRT}-\mathrm{PCR}$, quantitative real-time polymerase chain reaction.

that downregulation of miR-135a and upregulation of KIFC1 exist in GC, which were completely consistent with the experimental results of $\mathrm{Wu}$ et $\mathrm{al}^{26}$ and Oue et al. ${ }^{27} \mathrm{We}$ further investigated the effects of KIFC1 and miR-135a on GC cell proliferation and apoptosis and found that knockdown of KIFC1 inhibits proliferation and promotes apoptosis of GC cells, while the effect of miR-135a mimics on GC cell proliferation and apoptosis is consistent. Luciferase assay confirmed that miR-135a targets KIFC1 directly. An inverse correlation is also found between miR-135a and KIFC1 in 

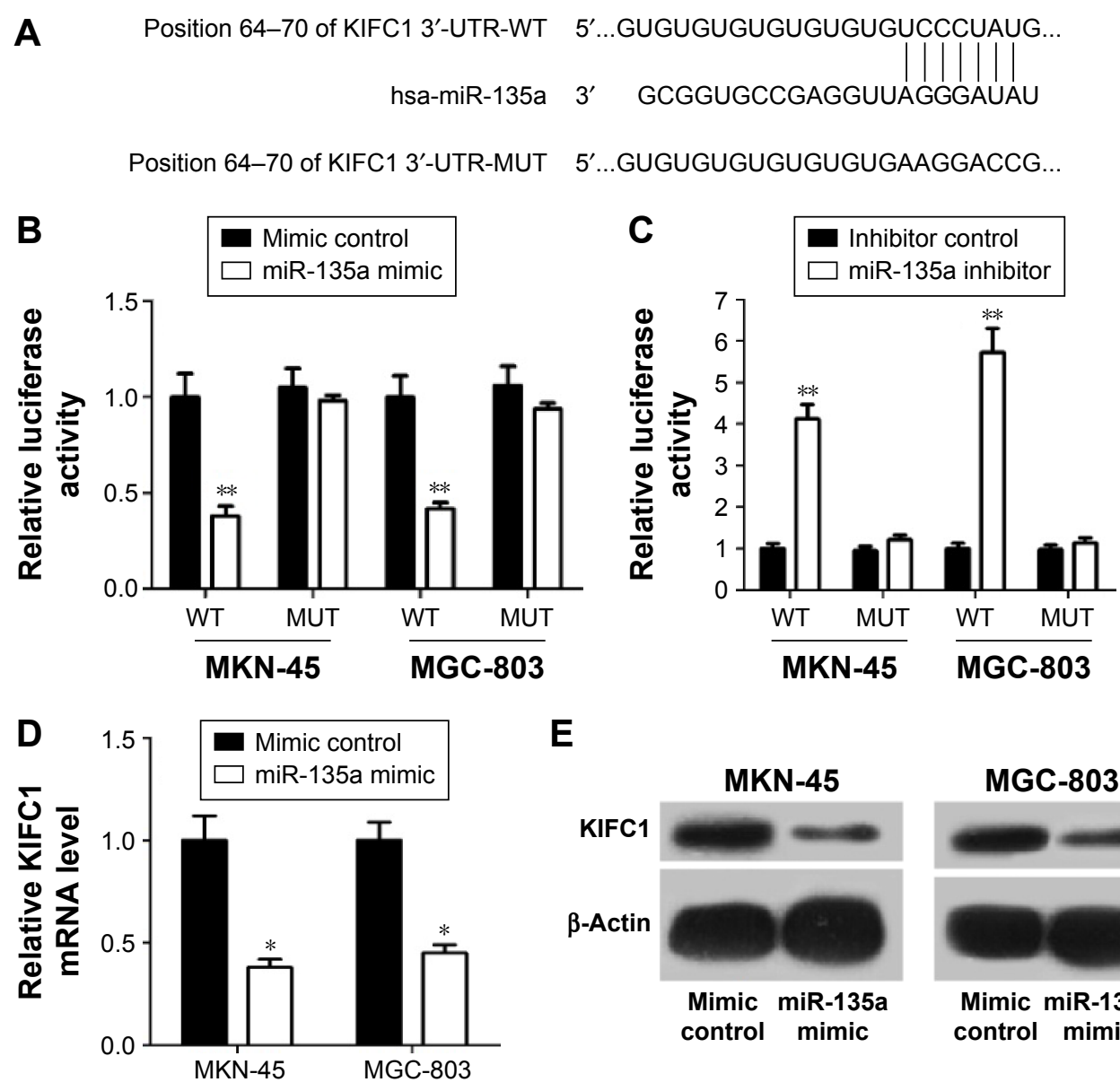

E
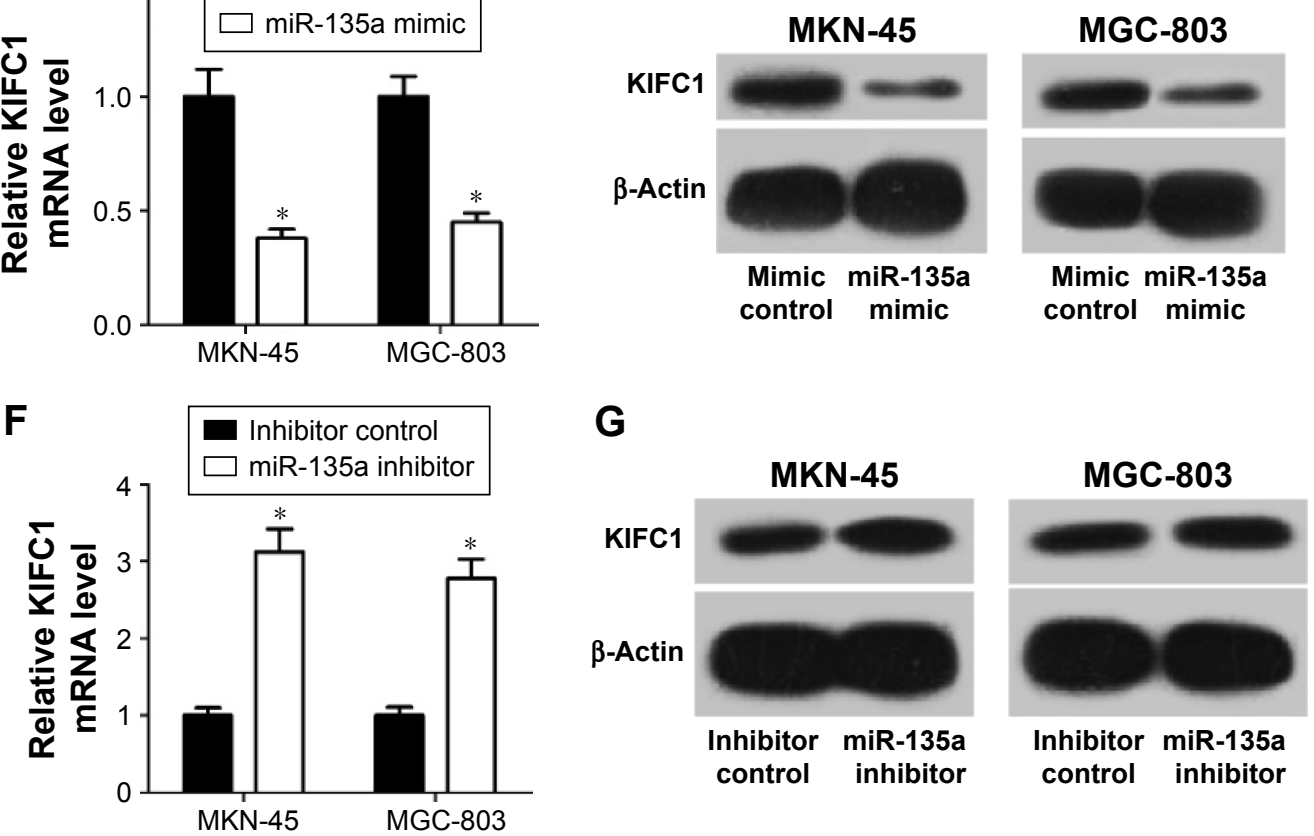

Figure $4 \mathrm{KIFCl}$ is a direct target of miR-135a.

Notes: (A) Bioinformatics-based target prediction analysis shows that KIFCI is a potential target of miR-I35a. (B) Luciferase reporter assay shows that MKN-45 and MGC-803 cells cotransfected with plasmid containing 3'-UTR-WT regions of KIFCI and miR-I35a mimics have significantly less luciferase activity than their controls, while mutation of the potential miR-135a binding sites in the KIFCI $3^{\prime}$-UTR abolishes this effect. (C) Luciferase reporter assay shows that MKN-45 and MGC-803 cells cotransfected with plasmid containing $3^{\prime}$-UTR-WT regions of KIFCI and miR-I35a inhibitors have significantly higher luciferase activity than their controls, which is abrogated by mutating the tentative miR-I35a binding sites in KIFCI. (D and E) qRT-PCR and Western blot assays show that overexpression of miR-I35a inhibits KIFCI mRNA and protein expression in MKN-45 and MGC-803 cells. (F and G) qRT-PCR and Western blot assays show that downregulation of miR-I35a enhances KIFCI mRNA and protein expression in MKN-45 and MGC-803 cells. $* P<0.05$ and $* * P<0.01$.

Abbreviations: mRNA, messenger RNA; MUT, mutant; qRT-PCR, quantitative real-time polymerase chain reaction; UTR, untranslated region; WT, wild-type.

MKN-45 and MGC-803 cells. These results confirmed our speculation that miR-135a regulates GC cell proliferation and apoptosis at least in part by targeting KIFC1.

\section{Conclusion}

In summary, downregulation of miR-135a and upregulation of KIFC1 exist in GC tissues and cell lines, and knockdown of KIFC1 inhibits proliferation and promotes apoptosis of GC cells, while miR-135a mimics have similar effects on GC cell proliferation and apoptosis. We provided evidence to verify that KIFC1 is a direct target of miR-135a which confirmed our speculation that the functional effect of miR-135a on GC cells at least in part depends on KIFC1. These findings suggest that miR-135a has an important role in the suppression 
of GC and presents a novel mechanism of miRNA-mediated KIFC1 expression in cancer cells.

\section{Disclosure}

The authors report no conflicts of interest in this work.

\section{References}

1. Ferlay J, Soerjomataram I, Dikshit R, et al. Cancer incidence and mortality worldwide: sources, methods and major patterns in GLOBOCAN 2012. Int J Cancer. 2015;136(5):E359-E386.

2. Torre LA, Bray F, Siegel RL, Ferlay J, Lortet-Tieulent J, Jemal A. Global cancer statistics, 2012. CA: Cancer J Clin. 2015;65(2):87-108.

3. Jiang Y, Ajani JA. Multidisciplinary management of gastric cancer. Curr Opin Gastroenterol. 2010;26(6):640-646.

4. Bartel DP. MicroRNAs: genomics, biogenesis, mechanism, and function. Cell. 2004;116(2):281-297.

5. Xiao B, Zhu E-D, Li N, et al. Increased miR-146a in gastric cancer directly targets SMAD4 and is involved in modulating cell proliferation and apoptosis. Oncol Rep. 2012;27(2):559-566.

6. Asangani I, Rasheed S, Nikolova D, et al. MicroRNA-21 (miR-21) posttranscriptionally downregulates tumor suppressor Pdcd4 and stimulates invasion, intravasation and metastasis in colorectal cancer. Oncogene. 2008;27(15):2128-2136

7. Okuda H, Xing F, Pandey PR, et al. miR-7 suppresses brain metastasis of breast cancer stem-like cells by modulating KLF4. Cancer Res. 2013; 73(4):1434-1444.

8. Chen HY, Lin YM, Chung HC, et al. miR-103/107 promote metastasis of colorectal cancer by targeting the metastasis suppressors DAPK and KLF4. Cancer Res. 2012;72(14):3631-3641.

9. Li L, Yuan L, Luo J, Gao J, Guo J, Xie X. MiR-34a inhibits proliferation and migration of breast cancer through down-regulation of Bcl-2 and SIRT1. Clin Exp Med. 2013;13(2):109-117.

10. Kinoshita T, Hanazawa T, Nohata N, et al. Tumor suppressive microRNA-218 inhibits cancer cell migration and invasion through targeting laminin-332 in head and neck squamous cell carcinoma. Oncotarget. 2012;3(11):1386-1400.

11. Tie J, Pan Y, Zhao L, et al. MiR-218 inhibits invasion and metastasis of gastric cancer by targeting the Robo1 receptor. PLoS Genet. 2010; 6(3):e1000879.

12. Ding L, Xu Y, Zhang W, et al. MiR-375 frequently downregulated in gastric cancer inhibits cell proliferation by targeting JAK2. Cell Res. 2010;20(7):784-793

13. Wu Q, Jin H, Yang Z, et al. MiR-150 promotes gastric cancer proliferation by negatively regulating the pro-apoptotic gene EGR2. Biochem Biophys Res Commun. 2010;392(3):340-345.
14. Chen D, Zhang D, Lu Y, et al. microRNA-217 inhibits tumor progression and metastasis by downregulating EZH2 and predicts favorable prognosis in gastric cancer. Oncotarget. 2015;6(13):10868-10879.

15. Liu S, Guo W, Shi J, et al. MicroRNA-135a contributes to the development of portal vein tumor thrombus by promoting metastasis in hepatocellular carcinoma. J Hepatol. 2012;56(2):389-396.

16. Mao XP, Zhang LS, Huang B, et al. Mir-135a enhances cellular proliferation through post-transcriptionally regulating PHLPP2 and FOXO1 in human bladder cancer. J Transl Med. 2015;13(1):86.

17. Zhou W, Li X, Liu F, et al. MiR-135a promotes growth and invasion of colorectal cancer via metastasis suppressor 1 in vitro. Acta Biochim Biophys Sin. 2012;44(10):838-846.

18. Wu S, Lin Y, Xu D, et al. MiR-135a functions as a selective killer of malignant glioma. Oncogene. 2012;31(34):3866-3874.

19. Tang W, Jiang Y, Mu X, Xu L, Cheng W, Wang X. MiR-135a functions as a tumor suppressor in epithelial ovarian cancer and regulates HOXA10 expression. Cell Signal. 2014;26(7):1420-1426.

20. Yamada Y, Hidaka H, Seki N, et al. Tumor-suppressive microRNA-135a inhibits cancer cell proliferation by targeting the c-MYC oncogene in renal cell carcinoma. Cancer Sci. 2013;104(3):304-312.

21. Shi H, Ji Y, Zhang D, Liu Y, Fang P. MiR-135a inhibits migration and invasion and regulates EMT-related marker genes by targeting KLF8 in lung cancer cells. Biochem Biophys Res Commun. 2015;465(1): $125-130$.

22. Pan HW, Li SC, Tsai KW. MicroRNA dysregulation in gastric cancer Curr Pharm Des. 2013;19(7):1273-1284.

23. Pawar S, Donthamsetty S, Pannu V, et al. KIFCI, a novel putative prognostic biomarker for ovarian adenocarcinomas: delineating protein interaction networks and signaling circuitries. $J$ Ovarian Res. 2014; $7(1): 1-9$.

24. Grinberg-Rashi H, Ofek E, Perelman M, et al. The expression of three genes in primary non-small cell lung cancer is associated with metastatic spread to the brain. Clin Cancer Res. 2009;15(5):1755-1761.

25. Li Y, Lu W, Chen D, et al. KIFC1 is a novel potential therapeutic target for breast cancer. Cancer Biol Ther. 2015;16(9):1316-1322.

26. Wu H, Huang M, Cao P, Wang T, Shu Y, Liu P. MiR-135a targets JAK2 and inhibits gastric cancer cell proliferation. Cancer Biol Ther. 2012; 13(5):281-288.

27. Oue N, Mukai S, Sentani K, Pham TTB, Ito R, Yasui W. Significance of KIFC1 in spheroid formation of gastric cancer cells. Cancer Res. 2015; 75(15 Suppl):1413-1413.

28. Guggenheim DE, Shah MA. Gastric cancer epidemiology and risk factors. J Surg Oncol. 2013;107(3):230-236.
OncoTargets and Therapy

\section{Publish your work in this journal}

OncoTargets and Therapy is an international, peer-reviewed, open access journal focusing on the pathological basis of all cancers, potential targets for therapy and treatment protocols employed to improve the management of cancer patients. The journal also focuses on the impact of management programs and new therapeutic agents and protocols on

\section{Dovepress}

patient perspectives such as quality of life, adherence and satisfaction. The manuscript management system is completely online and includes a very quick and fair peer-review system, which is all easy to use. Visit http://www.dovepress.com/testimonials.php to read real quotes from published authors. 